\title{
THE LOCAL SHARP MAXIMAL FUNCTION AND BMO ON LOCALLY HOMOGENEOUS SPACES
}

\author{
Marco Bramanti and Maria Stella Fanciullo \\ Politecnico di Milano, Dipartimento di Matematica \\ Via Bonardi 9, 20133 Milano, Italy; marco.bramanti@polimi.it \\ Università di Catania, Dipartimento di Matematica e Informatica \\ Viale Andrea Doria 6, 95125 Catania, Italy; fanciullo@dmi.unict.it
}

\begin{abstract}
We prove a local version of Fefferman-Stein inequality for the local sharp maximal function, and a local version of John-Nirenberg inequality for locally BMO functions, in the framework of locally homogeneous spaces, in the sense of Bramanti-Zhu [3].
\end{abstract}

\section{Introduction}

Real analysis and the theory of singular integrals have been developed first in the Euclidean setting and then in more general contexts, in view of their applications to harmonic analysis, partial differential equations, and complex analysis. Around 1970 the theory of spaces of homogeneous type, that is quasi-metric doubling measure spaces, started to be systematically developed in the monograph by Coifman-Weiss [11] and was successfully applied to several fields. Much more recently, some problems arising from the quest of a-priori estimates for PDEs suggested that real analysis would be a more flexible and useful tool if its concepts and results were stated also in a local version. The meaning of this localization is, roughly speaking, the following: we want an abstract theory which, when applied to the concrete setting of a bounded domain $\Omega \subset \mathbf{R}^{n}$ endowed with a local quasidistance $\rho$ and a locally doubling measure $d \mu$ (typically, the Lebesgue measure), brings to integrals over metric balls $B_{r}(x)$ properly contained in $\Omega$, and never requires to compute integrals over sets of the kind $B_{r}(x) \cap \Omega$, as happens when we apply the standard theory of spaces of homogeneous type to a bounded domain $\Omega$. These versions, however, are not easily obtained $a$ posteriori from the well established theory; instead, they require a careful analysis which often poses nontrivial new problems.

For instance, global $L^{p}$ estimates for certain operators of Ornstein-Uhlenbeck type were proved in [2] using results from a theory of nondoubling spaces, developed in [1], which in particular applies to certain locally doubling spaces. Bramanti-Zhu in [3] developed instead a theory of singular and fractional integrals in locally doubling spaces; these results were applied in [5] to the proof of $L^{p}$ and Schauder estimates for nonvariational operators structured on Hörmander's vector fields.

The aim of this paper is to continue the theory of locally doubling spaces, as started in [3], with two main results: a local version of Fefferman-Stein's theorem regarding the sharp maximal function, and a local version of John-Nirenberg inequality about $B M O$ functions. The first of these results, in its Euclidean version, is a key ingredient of a novel approach to the proof of $L^{p}$ estimates for nonvariational elliptic and parabolic operators with possibly discontinuous coefficients, first devised

https://doi.org/10.5186/aasfm.2017.4229

2010 Mathematics Subject Classification: Primary 42B25; Secondary 42B35, 46E30.

Key words: Locally homogeneous space, local sharp maximal function, local BMO, FeffermanStein inequality, John-Nirenberg inequality. 
by Krylov in [20]; the present extension can open the way to the application of these techniques to more general differential operators, as shown in [4].

Comparison with the existent literature and main results. The "sharp maximal function" was introduced in the Euclidean context by Fefferman-Stein in [14], where the related $L^{p}$ inequality was proved. In spaces of homogeneous type, the sharp maximal function has been studied, and the related Fefferman-Stein inequality established, by Pradolini-Salinas [23], see also Grafakos-Liu-Yang [15] for a vector valued case. In some spaces of generalized homogeneous type, in the sense of [7], this operator has been introduced and studied by Lai in [21]. Local sharp maximal functions have been studied, both in the Euclidean context and in spaces of homogeneous type, by several authors (with different definitions), see for instance JawerthTorchinsky [17], Shi-Torchinsky [24]. Here we follow the approach of Iwaniec [16] who proves, in the Euclidean context, a version of local sharp maximal inequality. His proof relies on a clever adaptation of Calderón-Zygmund decomposition, and the striking fact is that this construction can be adapted quite naturally to the abstract context of locally homogeneous spaces, exploiting the properties of the "dyadic cubes" abstractly constructed in this framework in [3]. Our first main result is the sharp maximal inequality stated in Theorem 3.4. This statement involves dyadic cubes and the dyadic local sharp maximal function (see Definition 3.3); since, however, these "cubes" are abstract objects which in the concrete application of the theory are not easily visualized, it is convenient to derive from Theorem 3.4 some consequences formulated in the language of metric balls and the local sharp maximal function (defined by means of balls, instead of dyadic cubes, see Definition 3.5). These results, more easily applicable, are Corollaries 3.7, 3.8, 3.9.

The space $B M O$ of functions with bounded mean oscillation was introduced in [18], where the famous "John-Nirenberg inequality" is proved. Versions of this space and this inequality in spaces of homogeneous type have been given by several authors, starting with Buckley [6] (see also Kronz [19], Caruso-Fanciullo [8] and Dafni-Yue [12]). To adapt this result to our context, we follow the approach contained in Mateu, Mattila, Nicolau, Orobitg [22, Appendix], see also Castillo, Ramos Fernández, Trousselot [9]. Our main result is Theorem 4.2, with its useful consequence, Theorem 4.5, stating that we can equivalently compute the mean oscillation of a function or its $L^{p}$ version for any $p \in(1, \infty)$, always computing averages over small balls.

For both our main results, i.e., the sharp maximal inequality and John-Nirenberg theorem, we stress that our setting, namely that of locally doubling spaces, is different from those considered in all the aforementioned bibliography.

Plan of the paper. Section 2 contains some basic facts about locally doubling spaces; in Section 3 the local sharp maximal function is studied and several $L^{p}$ inequalities are proved about it; in section 4 the local John-Nirenberg inequality is proved.

\section{Preliminaries about locally homogeneous spaces}

We start recalling the abstract context of locally homogeneous spaces, as introduced in [3].

(H1) Let $\Omega$ be a set, endowed with a function $\rho: \Omega \times \Omega \rightarrow[0, \infty)$ such that for any $x, y \in \Omega$ :

(a) $\rho(x, y)=0 \Longleftrightarrow x=y$;

(b) $\rho(x, y)=\rho(y, x)$. 
For any $x \in \Omega, r>0$, let us define the ball

$$
B(x, r)=\{y \in \Omega: \rho(x, y)<r\} .
$$

These balls can be used to define a topology in $\Omega$, saying that $A \subset \Omega$ is open if for any $x \in A$ there exists $r>0$ such that $B(x, r) \subset A$. Also, we will say that $E \subset \Omega$ is bounded if $E$ is contained in some ball.

Let us assume that:

(H2) (a) the balls are open with respect to this topology;

(b) for any $x \in \Omega$ and $r>0$ the closure of $B(x, r)$ is contained in $\{y \in \Omega$ : $\rho(x, y) \leq r\}$.

It can be proved (see [3, Prop. 2.4]) that the validity of conditions (H2) (a) and (b) is equivalent to the following:

(H2') $\rho(x, y)$ is a continuous function of $x$ for any fixed $y \in \Omega$.

(H3) Let $\mu$ be a positive regular Borel measure in $\Omega$.

(H4) Assume there exists an increasing sequence $\left\{\Omega_{n}\right\}_{n=1}^{\infty}$ of bounded measurable subsets of $\Omega$, such that

$$
\bigcup_{n=1}^{\infty} \Omega_{n}=\Omega
$$

and such for, any $n=1,2,3, \ldots$

(i) the closure of $\Omega_{n}$ in $\Omega$ is compact;

(ii) there exists $\varepsilon_{n}>0$ such that

$$
\left\{x \in \Omega: \rho(x, y)<2 \varepsilon_{n} \text { for some } y \in \Omega_{n}\right\} \subset \Omega_{n+1} ;
$$

We also assume that:

(H5) there exists $B_{n} \geq 1$ such that for any $x, y, z \in \Omega_{n}$

$$
\rho(x, y) \leq B_{n}(\rho(x, z)+\rho(z, y)) ;
$$

(H6) there exists $C_{n}>1$ such that for any $x \in \Omega_{n}, 0<r \leq \varepsilon_{n}$ we have

$$
0<\mu(B(x, 2 r)) \leq C_{n} \mu(B(x, r))<\infty .
$$

(Note that for $x \in \Omega_{n}$ and $r \leq \varepsilon_{n}$ we also have $\left.B(x, 2 r) \subset \Omega_{n+1}\right)$.

Definition 2.1. We will say that $\left(\Omega,\left\{\Omega_{n}\right\}_{n=1}^{\infty}, \rho, \mu\right)$ is a locally homogeneous space if assumptions (H1) to (H6) hold.

Dependence on the constants. The numbers $\varepsilon_{n}, B_{n}, C_{n}$ will be called "the constants of $\Omega_{n}$ ". It is not restrictive to assume that $B_{n}, C_{n}$ are nondecreasing sequences, and $\varepsilon_{n}$ is a nonincreasing sequence. Throughout the paper our estimates, for a fixed $\Omega_{n}$, will often depend not only on the constants of $\Omega_{n}$, but also (possibly) on the constants of $\Omega_{n+1}, \Omega_{n+2}, \Omega_{n+3}$. We will briefly say that "a constant depends on $n$ " to mean this type of dependence.

In the language of [11], $\rho$ is a quasidistance in each set $\Omega_{n}$; we can also say that it is a local quasidistance in $\Omega$. We stress that the two conditions appearing in (H2) are logically independent each from the other, and they do not follow from (2.3), even when $\rho$ is a quasidistance in $\Omega$, that is when $B_{n}=B>1$ for all $n$. If, however, $\rho$ is a distance in $\Omega$, that is $B_{n}=1$ for all $n$, then (H2) is automatically fulfilled. The continuity of $\rho$ also implies that (2.3) still holds for $x, y, z \in \bar{\Omega}_{n}$. Also, note that $\mu\left(\Omega_{n}\right)<\infty$ for every $n$, since $\bar{\Omega}_{n}$ is compact.

The basic concepts about Vitali covering lemma, the local maximal function and its $L^{p}$ bound can be easily adapted to this context: 
Lemma 2.2. (Vitali covering Lemma) Let $E$ be a measurable subset of $\Omega_{n}$ and let $\left\{B\left(x_{\lambda}, r_{\lambda}\right)\right\}_{\lambda \in \Lambda}$ be a family of balls with centers $x_{\lambda} \in \Omega_{n}$ and radii $0<r_{\lambda} \leq$ $r_{n} \equiv 2 \varepsilon_{n} /\left(2 B_{n+1}+3 B_{n+1}^{2}\right)$, such that $E \subset \bigcup_{\lambda \in \Lambda} B\left(x_{\lambda}, r_{\lambda}\right)$. Then one can select a countable subcollection $\left\{B\left(x_{\lambda_{j}}, r_{\lambda_{j}}\right)\right\}_{j=1}^{\infty}$ of mutually disjoint balls so that

$$
E \subset \bigcup_{j=1}^{\infty} B\left(x_{\lambda_{j}}, K_{n} r_{\lambda_{j}}\right)
$$

with $K_{n}=\left(2 B_{n+1}+3 B_{n+1}^{2}\right)$ and, for some constant $c$ depending on $\Omega$,

$$
\sum_{j=1}^{\infty} \mu\left(B\left(x_{\lambda_{j}}, r_{\lambda_{j}}\right)\right) \geq c \mu(E) .
$$

We can then give the following

Definition 2.3. Fix $\Omega_{n}, \Omega_{n+1}$ and, for any $f \in L^{1}\left(\Omega_{n+1}\right)$ define the local maximal function

$$
M_{\Omega_{n}, \Omega_{n+1}} f(x)=\sup _{\substack{B(\bar{x}, r) \ni x \\ r \leq r_{n}}} \frac{1}{\mu(B(\bar{x}, r))} \int_{B(\bar{x}, r)}|f(y)| d \mu(y) \quad \text { for } x \in \Omega_{n}
$$

where $r_{n}=2 \varepsilon_{n} /\left(2 B_{n+1}+3 B_{n+1}^{2}\right)$ is the same number appearing in Vitali Lemma. (Actually, the following theorem still holds if this number $r_{n}$ is replaced by any smaller number).

Then (see [3, Thm. 8.3]):

Theorem 2.4. Let $f$ be a measurable function defined on $\Omega_{n+1}$. The following hold:

(a) If $f \in L^{p}\left(\Omega_{n+1}\right)$ for some $p \in[1, \infty]$, then $M_{\Omega_{n}, \Omega_{n+1}} f$ is finite almost everywhere in $\Omega_{n}$;

(b) if $f \in L^{1}\left(\Omega_{n+1}\right)$, then for every $t>0$,

$$
\mu\left(\left\{x \in \Omega_{n}:\left(M_{\Omega_{n}, \Omega_{n+1}} f\right)(x)>t\right\}\right) \leq \frac{c_{n}}{t} \int_{\Omega_{n+1}}|f(y)| d \mu(y) ;
$$

(c) if $f \in L^{p}\left(\Omega_{n+1}\right), 1<p \leq \infty$, then $M_{\Omega_{n}, \Omega_{n+1}} f \in L^{p}\left(\Omega_{n}\right)$ and

$$
\left\|M_{\Omega_{n}, \Omega_{n+1}} f\right\|_{L^{p}\left(\Omega_{n}\right)} \leq c_{n, p}\|f\|_{L^{p}\left(\Omega_{n+1}\right)} .
$$

By standard techniques, from the above theorem one can also prove the following:

Theorem 2.5. (Lebesgue differentiation theorem) For every $f \in L_{l o c}^{1}\left(\Omega_{n+1}\right)$ and a.e. $x \in \Omega_{n}$ there exists

$$
\lim _{r \rightarrow 0^{+}} \frac{1}{\mu(B(x, r))} \int_{B(x, r)} f(y) d \mu(y)=f(x) .
$$

In particular, for every $f \in L_{\mathrm{loc}}^{1}\left(\Omega_{n+1}\right)$ and a.e. $x \in \Omega_{n}$,

$$
|f(x)| \leq M_{\Omega_{n}, \Omega_{n+1}} f(x) .
$$

A deep construction which is carried out in [3, Thm. 8.3], adapting to our local context an analogous construction developed in doubling spaces by Christ [10] is that of dyadic cubes. Their relevant properties are collected in the following: 
Theorem 2.6. (Dyadic cubes, see [3, Thm. 3.1]) Let $\left(\Omega,\left\{\Omega_{n}\right\}_{n=1}^{\infty}, \rho, \mu\right)$ be a locally homogeneous space. For any $n=1,2,3, \ldots$ there exists a collection

$$
\Delta_{n}=\left\{Q_{\alpha}^{k} \subset \Omega, k=1,2,3, \ldots, \alpha \in I_{k}\right\}
$$

(where $I_{k}$ is a set of indices) of open sets called "dyadic cubes subordinated to $\Omega_{n}$ ", positive constants $a_{0}, c_{0}, c_{1}, c_{2}, \delta \in(0,1)$ and a set $E \subset \Omega_{n}$ of zero measure, such that for any $k=1,2,3, \ldots$, we have:

(a) $\forall \alpha \in I_{k}$, each $Q_{\alpha}^{k}$ contains a ball $B\left(z_{\alpha}^{k}, a_{0} \delta^{k}\right)$;

(b) $\bigcup_{\alpha \in I_{k}} Q_{\alpha}^{k} \subset \Omega_{n+1}$;

(c) $\forall \alpha \in I_{k}, 1 \leq l \leq k$, there exists $Q_{\beta}^{l} \supseteq Q_{\alpha}^{k}$;

(d) $\forall \alpha \in I_{k}$, diam $\left(Q_{\alpha}^{k}\right)<c_{1} \delta^{k}$ and $\overline{Q_{\alpha}^{k}} \subset B\left(z_{\alpha}^{k}, c_{1} \delta^{k}\right) \subset \Omega_{n+2}$;

(e) $\ell \geq k \Longrightarrow \forall \alpha \in I_{k}, \beta \in I_{l}, Q_{\beta}^{\ell} \subset Q_{\alpha}^{k}$ or $Q_{\beta}^{\ell} \cap Q_{\alpha}^{k}=\emptyset$;

(f) $\Omega_{n} \backslash \bigcup_{\alpha \in I_{k}} Q_{\alpha}^{k} \subset E$;

(g) $\forall \alpha \in I_{k}, x \in Q_{\alpha}^{k} \backslash E, j \geq 1$ there exists $Q_{\beta}^{j} \ni x$;

(h)

$$
\mu\left(B(x, 2 r) \cap Q_{\alpha}^{k}\right) \leq c_{2} \mu\left(B(x, r) \cap Q_{\alpha}^{k}\right)
$$

for any $x \in Q_{\alpha}^{k} \backslash E, r>0$. More precisely, for these $x$ and $r$ we have

$$
\mu\left(B(x, r) \cap Q_{\alpha}^{k}\right) \geq \begin{cases}c_{0} \mu(B(x, r)) & \text { for } r \leq \delta^{k}, \\ c_{0} \mu\left(Q_{\alpha}^{k}\right) & \text { for } r>\delta^{k}\end{cases}
$$

The sets $Q_{\alpha}^{k}$ can be thought as dyadic cubes of side length $\delta^{k}$. Note that $k$ is a positive integer, so we are only considering small dyadic cubes. The cubes $\left\{Q_{\alpha}^{k}\right\}$ are subordinated to a particular $\Omega_{n}$, meaning that they essentially cover $\Omega_{n}$ (that is, their union covers $\Omega_{n}$ up to a set of zero measure) and are contained in $\Omega_{n+1}$. Note that the cubes $Q_{\alpha}^{k}$ and all the constants depend on $n$, so we should write, more precisely

$$
\left\{Q_{\alpha}^{(n), k}\right\}_{\alpha \in I_{k}^{(n)}} ; \delta_{(n)} ; a_{0,(n)}, c_{0,(n)}, c_{1,(n)}, c_{2,(n)},
$$

but we will usually avoid this heavy notation.

In the proof of the above theorem, $\delta$ is chosen small enough, so it is not restrictive to assume $c_{1} \delta<2 \varepsilon_{n+1}$, which implies that the ball $B\left(z_{\alpha}^{k}, c_{1} \delta^{k}\right)$ appearing in point (d) is $\subset \Omega_{n+2}$. (We remark this fact because in [3] the inclusion $B\left(z_{\alpha}^{k}, c_{1} \delta^{k}\right) \subset \Omega_{n+2}$ is not stated).

Point (h) contains a crucial information: the triple $\left(Q_{\alpha}^{k}, \rho, d \mu\right)$ is a space of homogeneous type in the sense of Coifman-Weiss, that is the measure $\mu$ of $\rho$-balls restricted to $Q_{\alpha}^{k}$ is doubling. Note that in our context this property could fail to be true, instead, for the measure $\mu$ of $\rho$-balls restricted to a fixed $\rho$-ball.

We will also need the following:

Lemma 2.7. (Covering Lemma) For every $n$ and every positive integer $k$ large enough, the set $\Omega_{n}$ can be essentially covered by a finite union of dyadic cubes $Q_{\alpha}^{k}$ (subordinated to $\Omega_{n+1}$ ) with the following properties:

(i) $Q_{\alpha}^{k} \subset B\left(z_{\alpha}^{k}, c_{1} \delta^{k}\right) \subset \Omega_{n+1}$,

(ii) $B\left(z_{\alpha}^{k}, c_{1} \delta^{k}\right) \subset F_{\alpha}^{k}$ (essentially), $F_{\alpha}^{k} \subset B\left(z_{\alpha}^{k}, c^{\prime} \delta^{k}\right) \subset \Omega_{n+1}$, where the set $F_{\alpha}^{k}$ is a finite union of dyadic cubes $Q_{\beta_{\alpha}}^{k}$, hence $F_{\alpha}^{k}$ is a space of homogeneous type, that is satisfies (h) of the previous theorem.

Proof. Since the whole $\Omega_{n+1}$ can be essentially covered by the union of the dyadic cubes $Q_{\alpha}^{k}$ subordinated to $\Omega_{n+1}, \Omega_{n}$ is essentially covered by a subfamily of 
these. By $(\mathrm{d})$ in the previous theorem, for each $Q_{\alpha}^{k}$ of these cubes, there exists a ball $B\left(z_{\alpha}^{k}, c_{1} \delta^{k}\right)$ such that $Q_{\alpha}^{k} \subset B\left(z_{\alpha}^{k}, c_{1} \delta^{k}\right) \subset \Omega_{n+2}$. However, since $Q_{\alpha}^{k}$, and then $B\left(z_{\alpha}^{k}, c_{1} \delta^{k}\right)$, contains a point of $\Omega_{n}$, for $k$ large enough $B\left(z_{\alpha}^{k}, c_{1} \delta^{k}\right) \subset \Omega_{n+1}$, that is (i) holds.

Let $F_{\alpha}^{k}$ the union of all the dyadic cubes $Q_{\beta}^{k}$ intersecting $B\left(z_{\alpha}^{k}, c_{1} \delta^{k}\right)$. Since $B\left(z_{\alpha}^{k}, c_{1} \delta^{k}\right) \subset \Omega_{n+1}$ which is essentially covered by the union of all the dyadic cubes $Q_{\beta}^{k}$, then $B\left(z_{\alpha}^{k}, c_{1} \delta^{k}\right)$ is essentially covered by $F_{\alpha}^{k}$. Since, by (d) in the previous theorem, $\operatorname{diam}\left(Q_{\beta}^{k}\right)<c_{1} \delta^{k}$, and each $Q_{\beta}^{k}$ contained in $F_{\alpha}^{k}$ intersects $B\left(z_{\alpha}^{k}, c_{1} \delta^{k}\right)$, $\operatorname{diam} F_{\alpha}^{k}$ is comparable to $\delta^{k}$, so $F_{\alpha}^{k} \subset B\left(z_{\alpha}^{k}, c^{\prime} \delta^{k}\right)$, which is again contained in $\Omega_{n+1}$, for $k$ large enough, since the ball contains a point of $\Omega_{n}$. Finally, any finite union of dyadic cubes satisfies the doubling condition, by (2.7) and [3, Corollary 3.9].

\section{The local sharp maximal function}

As we have explained in the Introduction, the proof of the sharp maximal inequality will be achieved following the approach in [16], which exploits a suitable version of Calderón-Zygmund decomposition. We start proving in the context of locally homogeneous spaces the following decomposition lemma.

For any measurable set $E$ and function $f \in L^{1}(E)$, let

$$
f_{E}=\frac{1}{|E|} \int_{E} f
$$

Lemma 3.1. For fixed $\Omega_{n}, \Omega_{n+1}$ we consider the family $\Delta_{n}$ of dyadic cubes built in Theorem 2.6. Let $Q_{\alpha_{1}}^{1}$ be a fixed dyadic cube ("of first generation") and let $f \in$ $L^{1}\left(Q_{\alpha_{1}}^{1}\right)$. For any $\lambda \geq a \equiv|f|_{Q_{\alpha_{1}}^{1}}$ there exists a countable family $\mathcal{C}_{\lambda}=\left\{Q_{\lambda, j}\right\}_{j=1,2, \ldots}$ of pairwise disjoint dyadic subcubes of $Q_{\alpha_{1}}^{1}$ such that:

(i) $\lambda<|f|_{Q_{\lambda, j}} \leq c_{n} \lambda$ for $j=1,2, \ldots$;

(ii) if $\lambda \geq \mu \geq a$ then each cube $Q_{\lambda, j}$ is a subcube of one from the family $\mathcal{C}_{\mu}$;

(iii) $|f(x)| \leq \lambda$ for a.e. $x \in Q_{\alpha_{1}}^{1} \backslash \bigcup_{j} Q_{\lambda, j}$;

(iv) $\sum_{j}\left|Q_{\lambda, j}\right| \leq\left|\left\{x \in Q_{\alpha_{1}}^{1}: M f(x)>\frac{\lambda}{c_{n}^{\prime}}\right\}\right|$;

(v) $\left|\left\{x \in Q_{\alpha_{1}}^{1}: M f(x)>c_{n}^{\prime \prime} \lambda\right\}\right| \leq c_{n}^{\prime \prime \prime} \sum_{j}\left|Q_{\lambda, j}\right|$

where $c_{n}, c_{n}^{\prime}, c_{n}^{\prime \prime}, c_{n}^{\prime \prime \prime}$ are constants $>1$ only depending on $n$ and we let for simplicity

$$
M f=M_{\Omega_{n+1}, \Omega_{n+2}}\left(f \chi_{Q_{\alpha_{1}}^{1}}\right)
$$

(i.e., the local maximal function is computed after extending $f$ to zero outside $Q_{\alpha_{1}}^{1}$ ).

Remark 3.2. As will be apparent from the proof, this lemma still holds if instead of a fixed dyadic cube $Q_{\alpha_{1}}^{1}$ of the first generation we fix a cube $Q_{\alpha_{k_{0}}}^{k_{0}}$ of some fixed generation $k_{0}>1$. Throughout this section we will stick to the convention of considering $Q_{\alpha_{1}}^{1}$ a cube of first generation, just to simplify notation, however we must keep in mind that the results still hold under the more general assumption on $Q_{\alpha_{k_{0}}}^{k_{0}}$. Or, saying this with other words, we can think that the cube $Q_{\alpha_{1}}^{1}$ appearing in Theorem 3.4 and Corollaries 3.7 and 3.8 has diameter as small as we want.

Proof. By point (c) in Thm. 2.6 for every dyadic cube $Q \subset Q_{\alpha_{1}}^{1}$ there exists an increasing chain of dyadic cubes

$$
Q=Q_{\alpha_{k}}^{k} \subset Q_{\alpha_{k-1}}^{k-1} \subset \ldots \subset Q_{\alpha_{1}}^{1}
$$


For a fixed $\lambda \geq a=|f|_{Q_{\alpha_{1}}^{1}}$, in order to define the family $\mathcal{C}_{\lambda}$ we say that $Q \in \mathcal{C}_{\lambda}$ if, with the notation (3.1),

$$
\lambda<|f|_{Q} \text { and }|f|_{Q_{\alpha_{s}}^{s}} \leq \lambda \text { for } s=1,2, \ldots, k-1 .
$$

Note that any two cubes in $\mathcal{C}_{\lambda}$ are disjoint, otherwise by point (e) in Thm. 2.6 one should be contained in the other, so they would be two different steps in the same chain (3.1), which contradicts our rule of choice. Let us show that $\mathcal{C}_{\lambda}$ satisfies properties (i)-(iv).

(i). For $Q=Q_{\alpha_{k}}^{k} \in \mathcal{C}_{\lambda}$, by construction, $\lambda<|f|_{Q}$ and $|f|_{Q_{\alpha_{k-1}}^{k-1}} \leq \lambda$, hence

$$
|f|_{Q} \leq \frac{1}{\left|Q_{\alpha_{k}}^{k}\right|} \int_{Q_{\alpha_{k-1}}^{k-1}}|f| \leq \frac{\left|Q_{\alpha_{k-1}}^{k-1}\right|}{\left|Q_{\alpha_{k}}^{k}\right|}|f|_{Q_{\alpha_{k-1}}^{k-1}} \leq c_{n} \lambda
$$

since by points (a) and (d) in Thm. 2.6,

$$
B\left(z_{\alpha}^{k}, a_{0} \delta^{k}\right) \subset Q_{\alpha_{k}}^{k} \subset Q_{\alpha_{k-1}}^{k-1} \subset B\left(z_{\alpha_{k-1}}^{k-1}, c_{1} \delta^{k-1}\right),
$$

hence by the locally doubling condition

$$
\frac{\left|Q_{\alpha_{k-1}}^{k-1}\right|}{\left|Q_{\alpha_{k}}^{k}\right|} \leq c_{n}
$$

for some $c_{n}$ only depending on $n$ (in particular, independent of $k$ ). Hence (i) is proved.

(ii). For $Q=Q_{\alpha_{k}}^{k} \in \mathcal{C}_{\lambda}, \lambda \geq \mu \geq a$ we have

$$
|f|_{Q_{\alpha_{k}}^{k}}>\lambda \geq \mu
$$

hence in the chain (3.1) there is an $l$ such that $|f|_{Q_{\alpha_{l}}^{l}}>\mu,|f|_{Q_{\alpha_{l-1}}^{l-1}} \leq \mu$. This means that in the chain (3.1) there is a cube $Q^{\prime}=Q_{\alpha_{l}}^{l} \in \mathcal{C}_{\mu}$, and $Q^{\prime} \supset Q$.

(iii). Let $x \in Q_{\alpha_{1}}^{1} \backslash \bigcup_{j} Q_{\lambda, j}$ and let $Q$ be any dyadic cube such that $x \in Q \subset Q_{\alpha_{1}}^{1}$. Consider again the chain (3.1) starting with $Q$. By our choice of $x$, none of the cubes $Q_{\alpha_{l}}^{l}$ in this chain belongs to $\mathcal{C}_{\lambda}$, and this means that $|f|_{Q_{\alpha_{l}}^{l}} \leq \lambda$. Then by point $(\mathrm{g})$ in Thm. 2.6, for a.e. $x \in Q_{\alpha_{1}}^{1} \backslash \bigcup_{j} Q_{\lambda, j}$ there exists a decreasing sequence of dyadic cubes $\left\{Q_{\alpha_{l}}^{l}\right\}$ such that $|f|_{Q_{\alpha_{l}}^{l}} \leq \lambda$ and $\bigcap Q_{\alpha_{l}}^{l}=\{x\}$. By Lebesgue's differentiation theorem, (iii) follows.

(iv). Let

$$
f^{*}(x)=\sup _{x \in Q \in \Delta_{n}}|f|_{Q} .
$$

In the previous point we have proved that for a.e. $x \in Q_{\alpha_{1}}^{1} \backslash \bigcup_{j} Q_{\lambda, j}$ and dyadic cube $Q$ such that $x \in Q \subset Q_{\alpha_{1}}^{1}$, we have $|f|_{Q} \leq \lambda$. Hence

$$
f^{*}(x) \leq \lambda \text { for a.e. } x \in Q_{\alpha_{1}}^{1} \backslash \bigcup_{j} Q_{\lambda, j} .
$$

Conversely, if $x \in \bigcup_{j} Q_{\lambda, j}$ then $|f|_{Q_{\lambda, j}}>\lambda$ hence $f^{*}(x)>\lambda$. These two facts mean that, up to a set of zero measure,

$$
\bigcup_{j} Q_{\lambda, j}=\left\{x \in Q_{\alpha_{1}}^{1}: f^{*}(x)>\lambda\right\}
$$


hence, since the $\left\{Q_{\lambda, j}\right\}_{j}$ are pairwise disjoint,

$$
\sum_{j}\left|Q_{\lambda, j}\right|=\left|\left\{x \in Q_{\alpha_{1}}^{1}: f^{*}(x)>\lambda\right\}\right| .
$$

However, again by points (a) and (d) in Thm. 2.6 and the locally doubling condition,

$$
f^{*}(x) \leq c_{n} M_{\Omega_{n+1}, \Omega_{n+2}}\left(f \chi_{Q_{\alpha_{1}}^{1}}\right)(x) \equiv c_{n} M f(x),
$$

hence (iv) follows.

(v). Let $Q_{\lambda, j} \in \mathcal{C}_{\lambda}$. For some $k=2,3, \ldots$, we will have $Q_{\lambda, j}=Q_{\alpha_{k}}^{k}$ and by points (a) and (d) in Thm. 2.6,

$$
B\left(z_{\alpha}^{k}, a_{0} \delta^{k}\right) \subset Q_{\alpha_{k}}^{k} \subset B\left(z_{\alpha_{k}}^{k}, c_{1} \delta^{k}\right)
$$

for some $z_{\alpha}^{k}$. For a $K>1$ to be chosen later, let $K Q_{\lambda, j}=B\left(z_{\alpha_{k}}^{k}, K c_{1} \delta^{k}\right)$. For any $x \notin \bigcup_{j} K Q_{\lambda, j}$ and any ball $B=B_{r}(\bar{x})$ such that $x \in B_{r}(\bar{x})$ and $r \leq r_{0}$ we have, extending $f$ to zero outside $Q_{\alpha_{1}}^{1}$ if $B \varsubsetneqq Q_{\alpha_{1}}^{1}$,

$$
\int_{B}|f|=\int_{B \backslash \bigcup_{j} Q_{\lambda, j}}|f|+\sum_{j} \int_{B \cap Q_{\lambda, j}}|f|
$$

by point (iii)

$$
\leq \lambda|B|+\sum_{j: B \cap Q_{\lambda, j} \neq \emptyset} \int_{Q_{\lambda, j}}|f|
$$

by point (i)

$$
\leq \lambda|B|+c_{n} \lambda \sum_{j: B \cap Q_{\lambda, j} \neq \emptyset}\left|Q_{\lambda, j}\right|
$$

Next, we need the following

Claim. There exist $K, H>1$ (only depending on $n$ ) such that if $x \notin \bigcup_{i} K Q_{\lambda, i}$ and $B_{r}(\bar{x}) \cap Q_{\lambda, j} \neq \emptyset$, then $Q_{\lambda, j} \subset B_{H r}(\bar{x})$.

Proof of the Claim. Recall that

$$
\begin{gathered}
B\left(z_{\alpha}^{k}, a_{0} \delta^{k}\right) \subset Q_{\lambda, j} \subset B\left(z_{\alpha_{k}}^{k}, c_{1} \delta^{k}\right), \\
K Q_{\lambda, j}=B\left(z_{\alpha_{k}}^{k}, K c_{1} \delta^{k}\right) .
\end{gathered}
$$

Since $B_{r}(\bar{x}) \cap Q_{\lambda, j} \neq \emptyset$, in particular $B_{r}(\bar{x}) \cap B\left(z_{\alpha}^{k}, a_{0} \delta^{k}\right) \neq \emptyset$ hence

$$
\rho\left(\bar{x}, z_{\alpha}^{k}\right) \leq B_{n+1}\left(r+a_{0} \delta^{k}\right) .
$$

Since $x \in B_{r}(\bar{x})$,

$$
\rho\left(x, z_{\alpha}^{k}\right) \leq B_{n+2}\left(r+\rho\left(\bar{x}, z_{\alpha}^{k}\right)\right) \leq B_{n+2}\left(r+B_{n+1}\left(r+a_{0} \delta^{k}\right)\right)
$$

and since $x \notin K Q_{\lambda, j}$,

$$
B_{n+2}\left(r+B_{n+1}\left(r+a_{0} \delta^{k}\right)\right)>K c_{1} \delta^{k}
$$

which, picking $K=\frac{2 B_{n+1} B_{n+2} a_{0}}{c_{1}}$, gives

$$
\delta^{k}<\frac{\left(1+B_{n+1}\right)}{B_{n+1} a_{0}} r .
$$


Then $Q_{\lambda, j} \subset B\left(z_{\alpha_{k}}^{k}, c_{1} \delta^{k}\right) \subset B_{H r}(\bar{x})$ for a suitable $H$ depending on $n$, namely for $z \in Q_{\lambda, j}$

$$
\begin{aligned}
\rho(z, \bar{x}) & \leq B_{n+1}\left(c_{1} \delta^{k}+\rho\left(\bar{x}, z_{\alpha}^{k}\right)\right) \leq B_{n+1}\left(c_{1} \delta^{k}+B_{n+1}\left(r+a_{0} \delta^{k}\right)\right) \\
& \leq \delta^{k}\left(B_{n+1} c_{1}+B_{n+1}^{2} a_{0}\right)+B_{n+1}^{2} r \\
& \leq \frac{\left(1+B_{n+1}\right)}{B_{n+1} a_{0}} r\left(B_{n+1} c_{1}+B_{n+1}^{2} a_{0}\right)+B_{n+1}^{2} r \equiv H r
\end{aligned}
$$

which proves the Claim.

Let us come back to the proof of (v). By (3.2) and the Claim we have (since the dyadic cubes in the sum are disjoint)

$$
\int_{B_{r}(\bar{x})}|f| \leq \lambda\left|B_{r}(\bar{x})\right|+c_{n} \lambda\left|B_{H r}(\bar{x})\right|
$$

and, by the locally doubling condition, for $r \leq r_{n}$ small enough,

$$
|f|_{B} \leq c_{n}^{\prime} \lambda
$$

for every $B \ni x \in Q_{\alpha_{1}}^{1} \backslash \bigcup_{j} K Q_{\lambda, j}$, that is

$$
M f(x) \leq c_{n}^{\prime} \lambda
$$

for any such $x$, so that

$$
\left\{x \in Q_{\alpha_{1}}^{1}: M f(x)>c_{n}^{\prime} \lambda\right\} \subset \bigcup_{j} K Q_{\lambda, j}
$$

and

$$
\left|\left\{x \in Q_{\alpha_{1}}^{1}: M f(x)>c_{n}^{\prime} \lambda\right\}\right| \leq \sum_{j}\left|K Q_{\lambda, j}\right| \leq c_{n}^{\prime \prime} \sum_{j}\left|Q_{\lambda, j}\right| .
$$

We can now prove the following local analog of Fefferman-Stein inequality. Let us first define a version of dyadic sharp maximal function:

Definition 3.3. For $f \in L^{1}\left(Q_{\alpha_{1}}^{1}\right), x \in Q_{\alpha_{1}}^{1}$, let

$$
f_{\Delta}^{\#}(x)=\sup _{\substack{x \ni Q \in \Delta_{n} \\ Q \subset Q_{\alpha_{1}}^{1}}} \frac{1}{|Q|} \int_{Q}\left|f-f_{Q}\right|
$$

Note that this definition involves only the values of $f$ in $Q_{\alpha_{1}}^{1}$ (there is no need of extending $f$ outside that cube).

Theorem 3.4. (Local Fefferman-Stein inequality) Let $f \in L^{1}\left(Q_{\alpha_{1}}^{1}\right)$ and assume $f_{\Delta}^{\#} \in L^{p}\left(Q_{\alpha_{1}}^{1}\right)$ for some $p \in[1,+\infty)$. Then $M f \in L^{p}\left(Q_{\alpha_{1}}^{1}\right)$ and

$$
\left(\frac{1}{\left|Q_{\alpha_{1}}^{1}\right|} \int_{Q_{\alpha_{1}}^{1}}(M f)^{p}\right)^{1 / p} \leq c_{n, p}\left\{\left(\frac{1}{\left|Q_{\alpha_{1}}^{1}\right|} \int_{Q_{\alpha_{1}}^{1}}\left(f_{\Delta}^{\#}\right)^{p}\right)^{1 / p}+\left(\frac{1}{\left|Q_{\alpha_{1}}^{1}\right|} \int_{Q_{\alpha_{1}}^{1}}|f|\right)\right\}
$$

for some constant $c_{n, p}$ only depending on $n, p$, where, as above,

$$
M f=M_{\Omega_{n+1}, \Omega_{n+2}}\left(f \chi_{Q_{\alpha_{1}}^{1}}\right) .
$$

Proof. Let $a=|f|_{Q_{\alpha_{1}}^{1}}$. We start proving the following estimate: for every $\lambda \geq 2 c_{n} a$ and every $A>0$,

$$
\sum_{j}\left|Q_{\lambda, j}\right| \leq\left|\left\{x \in Q_{\alpha_{1}}^{1}: f_{\Delta}^{\#}(x)>\frac{\lambda}{A}\right\}\right|+\frac{2}{A} \sum_{Q \in \mathcal{C}_{\lambda / 2 c_{n}}}|Q| .
$$


Fix $\lambda \geq 2 c_{n}$ a. By point (i) in Lemma 3.1, for any $Q_{\lambda, k} \in \mathcal{C}_{\lambda}$,

$$
\lambda<|f|_{Q_{\lambda, j}} \leq c_{n} \lambda
$$

also, for any $Q \in \mathcal{C}_{\lambda / 2 c_{n}}$,

so that, for such $Q_{\lambda, j}$ and $Q$,

$$
|f|_{Q} \leq c_{n} \frac{\lambda}{2 c_{n}}=\frac{\lambda}{2}
$$

$$
\begin{aligned}
\frac{1}{\left|Q_{\lambda, j}\right|} \int_{Q_{\lambda, j}}\left|f-f_{Q}\right| & \geq|f|_{Q_{\lambda, j}}-|f|_{Q}>\lambda-\frac{\lambda}{2}=\frac{\lambda}{2} \\
\left|Q_{\lambda, j}\right| & <\frac{2}{\lambda} \int_{Q_{\lambda, j}}\left|f-f_{Q}\right| .
\end{aligned}
$$

By point (ii) in Lemma 3.1, since $\lambda>\lambda / 2 c_{n}$, any $Q_{\lambda, j} \in \mathcal{C}_{\lambda}$ is contained in some $Q \in \mathcal{C}_{\lambda / 2 c_{n}}$; also, the cubes $Q \in \mathcal{C}_{\lambda / 2 c_{n}}$, like the cubes $Q_{\lambda, j} \in \mathcal{C}_{\lambda}$ are pairwise disjoint, hence we can write

$$
\sum_{j}\left|Q_{\lambda, j}\right|=\sum_{Q \in \mathcal{C}_{\lambda / 2 c_{n}}} \sum_{\substack{Q_{\lambda, j} \in \mathcal{C}_{\lambda} \\ Q_{\lambda, j} \subset Q}}\left|Q_{\lambda, j}\right| .
$$

For any $Q \in \mathcal{C}_{\lambda / 2 c_{n}}$, by (3.5) and since the $Q_{\lambda, j}$ are disjoint

$$
\sum_{\substack{Q_{\lambda, j} \in \mathcal{C}_{\lambda} \\ Q_{\lambda, j} \subset Q}}\left|Q_{\lambda, j}\right| \leq \sum_{\substack{Q_{\lambda, j} \in \mathcal{C}_{\lambda} \\ Q_{\lambda, j} \subset Q}} \frac{2}{\lambda} \int_{Q_{\lambda, j}}\left|f-f_{Q}\right| \leq \frac{2}{\lambda} \int_{Q}\left|f-f_{Q}\right| .
$$

Let now fix a number $A>0$ and distinguish two cases:

a. If $\frac{1}{|Q|} \int_{Q}\left|f-f_{Q}\right| \leq \frac{\lambda}{A}$, then

$$
\sum_{\substack{Q_{\lambda, j} \in \mathcal{C}_{\lambda} \\ Q_{\lambda, j} \subset Q}}\left|Q_{\lambda, j}\right| \leq \frac{2}{\lambda} \frac{\lambda}{A}|Q|=\frac{2}{A}|Q|
$$

b. If $\frac{1}{|Q|} \int_{Q}\left|f-f_{Q}\right|>\frac{\lambda}{A}$, then for every $x \in Q$

$$
f_{\Delta}^{\#}(x)>\frac{\lambda}{A},
$$

that is

$$
Q \subset\left\{x \in Q_{\alpha_{1}}^{1}: f_{\Delta}^{\#}(x)>\frac{\lambda}{A}\right\}
$$

and

$$
\sum_{\substack{Q_{\lambda, j} \in \mathcal{C}_{\lambda} \\ Q_{\lambda, j} \subset Q}}\left|Q_{\lambda, j}\right| \leq\left|Q \cap\left\{x \in Q_{\alpha_{1}}^{1}: f_{\Delta}^{\#}(x)>\frac{\lambda}{A}\right\}\right| .
$$

In any case we can write

$$
\sum_{\substack{Q_{\lambda, j} \in \mathcal{C}_{\lambda} \\ Q_{\lambda, j} \subset Q}}\left|Q_{\lambda, j}\right| \leq\left|Q \cap\left\{x \in Q_{\alpha_{1}}^{1}: f_{\Delta}^{\#}(x)>\frac{\lambda}{A}\right\}\right|+\frac{2}{A}|Q| .
$$

Adding up these inequalities for $Q \in \mathcal{C}_{\lambda / 2 c_{n}}$, recalling (3.6) and the fact that the cubes $Q$ are pairwise disjoint, we get (3.4). 
By (3.4) and points (v), (iv) in Lemma 3.1, we have

$$
\begin{aligned}
& \left|\left\{x \in Q_{\alpha_{1}}^{1}: M f(x)>c_{n}^{\prime \prime} \lambda\right\}\right| \\
& \leq c_{n}^{\prime \prime \prime}\left(\left|\left\{x \in Q_{\alpha_{1}}^{1}: f_{\Delta}^{\#}(x)>\frac{\lambda}{A}\right\}\right|+\frac{2}{A} \sum_{Q \in \mathcal{C}_{\lambda / 2 c_{n}}}|Q|\right) \\
& \leq c_{n}^{\prime \prime \prime}\left(\left|\left\{x \in Q_{\alpha_{1}}^{1}: f_{\Delta}^{\#}(x)>\frac{\lambda}{A}\right\}\right|+\frac{2}{A}\left|\left\{x \in Q_{\alpha_{1}}^{1}: M f(x)>\frac{\lambda}{2 c_{n} c_{n}^{\prime}}\right\}\right|\right)
\end{aligned}
$$

for any $A>0, \lambda \geq 2 c_{n} a$.

We now want to compute integrals using the identity (for any $F \in L^{p}\left(Q_{\alpha_{1}}^{1}\right), 1 \leq$ $p<\infty)$

\section{Letting}

$$
\int_{Q_{\alpha_{1}}^{1}}|F(y)|^{p} d y=\int_{0}^{+\infty} p t^{p-1}\left|\left\{x \in Q_{\alpha_{1}}^{1}:|F(x)|>t\right\}\right| d t
$$

$$
\mu(t)=\left|\left\{x \in Q_{\alpha_{1}}^{1}:|M f(x)|>t\right\}\right|
$$

and integrating (3.7) for $\lambda \in\left(2 c_{n} a, N\right)$ and any fixed $N>2 c_{n}|f|_{Q_{\alpha_{1}}^{1}}$ after multiplying by $p \lambda^{p-1}$ we have

$$
\begin{aligned}
\int_{2 c_{n} a}^{N} p \lambda^{p-1} \mu\left(c_{n}^{\prime \prime} \lambda\right) d \lambda \leq & c_{n}^{\prime \prime \prime}\left(\int_{2 c_{n} a}^{N} p \lambda^{p-1}\left|\left\{x \in Q_{\alpha_{1}}^{1}: f_{\Delta}^{\#}(x)>\frac{\lambda}{A}\right\}\right| d \lambda\right. \\
& \left.+\frac{2}{A} \int_{2 c_{n} a}^{N} p \lambda^{p-1} \mu\left(\frac{\lambda}{2 c_{n} c_{n}^{\prime}}\right) d \lambda\right) .
\end{aligned}
$$

Changing variable in each of the three integrals in (3.8) we get:

$$
\begin{aligned}
\int_{2 c_{n} c_{n}^{\prime \prime a}}^{c_{n}^{\prime \prime} N} p t^{p-1} \mu(t) d t \leq & \left(c_{n}^{\prime \prime}\right)^{p} c_{n}^{\prime \prime \prime}\left(A^{p} \int_{0}^{+\infty} p t^{p-1}\left|\left\{x \in Q_{\alpha_{1}}^{1}: f_{\Delta}^{\#}(x)>t\right\}\right| d t\right. \\
& \left.+\frac{2}{A}\left(2 c_{n} c_{n}^{\prime}\right)^{p} \int_{0}^{\frac{N}{2 c_{n} c_{n}^{\prime}}} p t^{p-1} \mu(t) d t\right) .
\end{aligned}
$$

Using also the elementary inequality

$$
\int_{0}^{2 c_{n} c_{n}^{\prime \prime} a} p t^{p-1} \mu(t) d t \leq \int_{0}^{2 c_{n} c_{n}^{\prime \prime} a} p t^{p-1}\left|Q_{\alpha_{1}}^{1}\right| d t=\left|Q_{\alpha_{1}}^{1}\right|\left(2 c_{n} c_{n}^{\prime \prime} a\right)^{p},
$$

together with (3.9), since $\frac{N}{2 c_{n} c_{n}^{\prime}}<N<c_{n}^{\prime \prime} N$ we get

$$
\begin{aligned}
\int_{0}^{c_{n}^{\prime \prime} N} p t^{p-1} \mu(t) d t \leq & \left(c_{n}^{\prime \prime}\right)^{p} c_{n}^{\prime \prime \prime}\left(A^{p} \int_{0}^{+\infty} p t^{p-1}\left|\left\{x \in Q_{\alpha_{1}}^{1}: f_{\Delta}^{\#}(x)>t\right\}\right| d t\right. \\
& \left.+\frac{2}{A}\left(2 c_{n} c_{n}^{\prime}\right)^{p} \int_{0}^{c_{n}^{\prime \prime} N} p t^{p-1} \mu(t) d t\right)+\left|Q_{\alpha_{1}}^{1}\right|\left(2 c_{n} c_{n}^{\prime \prime} a\right)^{p} .
\end{aligned}
$$

Letting finally $A=4\left(2 c_{n} c_{n}^{\prime} c_{n}^{\prime \prime}\right)^{p} c_{n}^{\prime \prime \prime}$ we conclude

$$
\int_{0}^{c_{n}^{\prime \prime} N} p t^{p-1} \mu(t) d t \leq c_{n, p}\left(\int_{0}^{+\infty} p t^{p-1}\left|\left\{x \in Q_{\alpha_{1}}^{1}: f_{\Delta}^{\#}(x)>t\right\}\right| d t+\left|Q_{\alpha_{1}}^{1}\right||f|_{Q_{\alpha_{1}}^{1}}^{p}\right)
$$

which implies that $M f \in L^{p}\left(Q_{\alpha_{1}}^{1}\right)$ and

$$
\|M f\|_{L^{p}\left(Q_{\alpha_{1}}^{1}\right)}^{p} \leq c_{n, p}\left(\left\|f_{\Delta}^{\#}\right\|_{L^{p}\left(Q_{\alpha_{1}}^{1}\right)}^{p}+\left|Q_{\alpha_{1}}^{1}\right||f|_{Q_{\alpha_{1}}^{1}}^{p}\right)
$$


that is $(3.3)$.

We now want to reformulate the above theorem in terms of balls, instead of dyadic cubes, to make it more easily applicable to concrete situations. This reformulation can be done in several ways. First of all, we introduce the local sharp maximal functions defined by balls instead of cubes.

Definition 3.5. For $f \in L_{l o c}^{1}\left(\Omega_{n+1}\right), x \in \Omega_{n}$, let

$$
f_{\Omega_{n}, \Omega_{n+1}}^{\#}(x)=\sup _{\substack{B(\bar{x}, r) \ni x \\ \bar{x} \in \Omega_{n}, r \leq \varepsilon_{n}}} \frac{1}{|B(\bar{x}, r)|} \int_{B(\bar{x}, r)}\left|f-f_{B(\bar{x}, r)}\right| .
$$

Let us compare this function with its dyadic version $f_{\Delta}^{\#}$ :

Lemma 3.6. With the above notation, for any $x \in Q_{\alpha_{1}}^{1}$,

$$
f_{\Delta}^{\#}(x) \leq c_{n} f_{\Omega_{n+1}, \Omega_{n+2}}^{\#}(x)
$$

for some constant $c_{n}$ only depending on $n$. Here the function $f$ can be assumed either in $L_{\text {loc }}^{1}\left(\Omega_{n+2}\right)$ or in $L^{1}\left(Q_{\alpha_{1}}^{1}\right)$ and extended to zero outside $Q_{\alpha_{1}}^{1}$.

Proof. For any dyadic cube $Q=Q_{\alpha_{k}}^{k} \subset Q_{\alpha_{1}}^{1} \subset \Omega_{n+1}$ we have (see points (a), (d) in Theorem 2.6)

$$
B\left(z_{\alpha}^{k}, a_{0} \delta^{k}\right) \subset Q \subset B\left(z_{\alpha_{k}}^{k}, c_{1} \delta^{k}\right) \subset \Omega_{n+2} .
$$

Let us briefly write $B_{1}, B_{2}$ in place of $B\left(z_{\alpha}^{k}, a_{0} \delta^{k}\right), B\left(z_{\alpha_{k}}^{k}, c_{1} \delta^{k}\right)$. Then by the locally doubling condition

$$
\frac{\left|B_{2}\right|}{|Q|} \leq \frac{\left|B_{2}\right|}{\left|B_{1}\right|} \leq c_{n}
$$

and we can write

$$
\begin{aligned}
\frac{1}{|Q|} \int_{Q}\left|f-f_{Q}\right| & \leq \frac{1}{|Q|} \int_{B_{2}}\left|f-f_{Q}\right| \leq c_{n} \frac{1}{\left|B_{2}\right|} \int_{B_{2}}\left|f-f_{Q}\right| \\
& \leq c_{n}\left(\frac{1}{\left|B_{2}\right|} \int_{B_{2}}\left|f-f_{B_{2}}\right|+\left|f_{Q}-f_{B_{2}}\right|\right) .
\end{aligned}
$$

Also,

$$
\left|f_{Q}-f_{B_{2}}\right|=\left|\frac{1}{|Q|} \int_{Q}\left(f-f_{B_{2}}\right)\right| \leq \frac{1}{|Q|} \int_{Q}\left|f-f_{B_{2}}\right| \leq c_{n} \frac{1}{\left|B_{2}\right|} \int_{B_{2}}\left|f-f_{B_{2}}\right|
$$

hence

$$
\frac{1}{|Q|} \int_{Q}\left|f-f_{Q}\right| \leq c_{n}\left(1+c_{n}\right) \frac{1}{\left|B_{2}\right|} \int_{B_{2}}\left|f-f_{B_{2}}\right|
$$

and the assertion follows.

Exploiting the previous Lemma and Theorem 2.5 we can now rewrite the statement of Theorem 3.4 as follows:

Corollary 3.7. Let $f \in L^{1}\left(Q_{\alpha_{1}}^{1}\right)$ and assume $f_{\Omega_{n+1}, \Omega_{n+2}}^{\#} \in L^{p}\left(Q_{\alpha_{1}}^{1}\right)$ for some $p \in[1,+\infty)$. Then $f \in L^{p}\left(Q_{\alpha_{1}}^{1}\right)$ and

$$
\left(\frac{1}{\left|Q_{\alpha_{1}}^{1}\right|} \int_{Q_{\alpha_{1}}^{1}}|M f|^{p}\right)^{1 / p} \leq c_{n, p}\left\{\left(\frac{1}{\left|Q_{\alpha_{1}}^{1}\right|} \int_{Q_{\alpha_{1}}^{1}}\left(f_{\Omega_{n+1}, \Omega_{n+2}}^{\#}\right)^{p}\right)^{1 / p}+\left(\frac{1}{\left|Q_{\alpha_{1}}^{1}\right|} \int_{Q_{\alpha_{1}}^{1}}|f|\right)\right\}
$$


for some constant $c_{n, p}$ only depending on $n, p$. Here $M$ is defined as in Theorem 3.4 and, again, the function $f$ can be assumed either in $L_{\mathrm{loc}}^{1}\left(\Omega_{n+2}\right)$ or in $L^{1}\left(Q_{\alpha_{1}}^{1}\right)$ and extended to zero outside $Q_{\alpha_{1}}^{1}$.

The following is also useful:

Corollary 3.8. Let $B_{1} \subset Q_{\alpha_{1}}^{1} \subset B_{2}$ with $B_{1}, B_{2}$ concentric balls of comparable radii (like in the proof of Lemma 3.6) and assume that $f \in L^{1}\left(B_{2}\right)$ with $f_{\Omega_{n+1}, \Omega_{n+2}}^{\#} \in$ $L^{p}\left(Q_{\alpha_{1}}^{1}\right)$ for some $p \in[1,+\infty)$ and $f_{B_{2}}=0$ (where the function $f$ can be assumed either in $L_{\text {loc }}^{1}\left(\Omega_{n+2}\right)$ or in $L^{1}\left(B_{2}\right)$ and extended to zero outside $\left.B_{2}\right)$. Then $f \in$ $L^{p}\left(Q_{\alpha_{1}}^{1}\right)$ and we have

$$
\begin{gathered}
\left(\frac{1}{\left|Q_{\alpha_{1}}^{1}\right|} \int_{Q_{\alpha_{1}}^{1}}|f|^{p}\right)^{1 / p} \leq c_{n, p}\left(\frac{1}{\left|Q_{\alpha_{1}}^{1}\right|} \int_{Q_{\alpha_{1}}^{1}}\left(f_{\Omega_{n+1}, \Omega_{n+2}}^{\#}\right)^{p}\right)^{1 / p}, \\
\left(\frac{1}{\left|B_{1}\right|} \int_{B_{1}}|f|^{p}\right)^{1 / p} \leq c_{n, p}^{\prime}\left(\frac{1}{\left|B_{2}\right|} \int_{B_{2}}\left(f_{\Omega_{n+1}, \Omega_{n+2}}^{\#}\right)^{p}\right)^{1 / p}
\end{gathered}
$$

for some constants $c_{n, p}, c_{n, p}^{\prime}$ only depending on $n, p$. Also, removing the assumption $f_{B_{2}}=0$ we can write

$$
\left(\frac{1}{\left|B_{1}\right|} \int_{B_{1}}\left|f-f_{B_{2}}\right|^{p}\right)^{1 / p} \leq c_{n, p}^{\prime}\left(\frac{1}{\left|B_{2}\right|} \int_{B_{2}}\left(f_{\Omega_{n+1}, \Omega_{n+2}}^{\#}\right)^{p}\right)^{1 / p} .
$$

Proof. We can write

$$
\frac{1}{\left|Q_{\alpha_{1}}^{1}\right|} \int_{Q_{\alpha_{1}}^{1}}|f|=\frac{1}{\left|Q_{\alpha_{1}}^{1}\right|} \int_{Q_{\alpha_{1}}^{1}}\left|f-f_{B_{2}}\right| \leq c_{n} \frac{1}{\left|B_{2}\right|} \int_{B_{2}}\left|f-f_{B_{2}}\right| \leq c_{n} f_{\Omega_{n+1}, \Omega_{n+2}}^{\#}(x)
$$

for every $x \in B_{2}$. Averaging this inequality on $Q_{\alpha_{1}}^{1}$ we get

$$
\frac{1}{\left|Q_{\alpha_{1}}^{1}\right|} \int_{Q_{\alpha_{1}}^{1}}|f| \leq c_{n} \frac{1}{\left|Q_{\alpha_{1}}^{1}\right|} \int_{Q_{\alpha_{1}}^{1}} f_{\Omega_{n+1}, \Omega_{n+2}}^{\#} \leq c_{n}\left(\frac{1}{\left|Q_{\alpha_{1}}^{1}\right|} \int_{Q_{\alpha_{1}}^{1}}\left(f_{\Omega_{n+1}, \Omega_{n+2}}^{\#}\right)^{p}\right)^{1 / p}
$$

so that, by recalling Corollary 3.7

$$
\left(\frac{1}{\left|Q_{\alpha_{1}}^{1}\right|} \int_{Q_{\alpha_{1}}^{1}}|f|^{p}\right)^{1 / p} \leq c_{n, p}\left(\frac{1}{\left|Q_{\alpha_{1}}^{1}\right|} \int_{Q_{\alpha_{1}}^{1}}\left(f_{\Omega_{n+1}, \Omega_{n+2}}^{\#}\right)^{p}\right)^{1 / p}
$$

which also implies the second inequality, by the locally doubling condition and the comparability of the radii of $B_{1}, B_{2}$.

Although, in the previous Corollary, the second inequality has the pleasant feature of involving balls instead of dyadic cubes (however, note the two different balls appearing at the left hand side of the last inequality), remember that we cannot choose these balls as we like, since they are related to dyadic cubes.

In concrete applications of this theory, we could use this result to bound $\|f\|_{L^{p}\left(\Omega_{n}\right)}$. To this aim, recall that the domain $\Omega_{n}$ can be covered (up to a zero measure set) by a finite union of dyadic cubes of the kind $Q_{\alpha_{1}}^{1}$, but $\Omega_{n}$ is not covered by the union of the corresponding smaller balls $B_{1}$. We then need to improve the previous corollary, replacing the dyadic cube $Q$ on the left hand side with a larger ball:

Corollary 3.9. For any $n$ and every $k$ large enough, the set $\Omega_{n}$ can be covered by a finite union of balls $B_{R}\left(x_{i}\right)$ of radii comparable to $\delta^{k}$ such that for any such ball 
$B_{R}$ and every $f$ supported in $B_{R}$ such that $f \in L^{1}\left(B_{R}\right), \int_{B_{R}} f=0$, and $f_{\Omega_{n+2}, \Omega_{n+3}}^{\#} \in$ $L_{l o c}^{p}\left(\Omega_{n+1}\right)$ for some $p \in[1, \infty)$, one has

$$
\|f\|_{L^{p}\left(B_{R}\right)} \leq c_{n, p}\left\|f_{\Omega_{n+2}, \Omega_{n+3}}^{\#}\right\|_{L^{p}\left(B_{\gamma R}\right)}
$$

with $\gamma>1$ absolute constant.

Proof. Applying Lemma 2.7, let us (essentially) cover $\Omega_{n}$ with a finite union of dyadic cubes

$$
Q_{\alpha}^{k} \subset B\left(z_{\alpha}^{k}, c_{1} \delta^{k}\right) \subset F_{\alpha}^{k} \subset B\left(z_{\alpha}^{k}, c^{\prime} \delta^{k}\right)
$$

(where the inclusion $B\left(z_{\alpha}^{k}, c_{1} \delta^{k}\right) \subset F_{\alpha}^{k}$ is only essential). We claim that the balls $B\left(z_{\alpha}^{k}, c_{1} \delta^{k}\right)$ are the required covering of $\Omega_{n}$. To see this, let $f$ be supported in $B\left(z_{\alpha}^{k}, c_{1} \delta^{k}\right)$ and with vanishing integral. Then the same is true for $f$ with respect to the larger ball $B\left(z_{\alpha}^{k}, c^{\prime} \delta^{k}\right)$. We can then apply Corollary 3.8 to each dyadic cube $Q_{\beta}^{k}$ which constitutes $F_{\alpha}^{k}$, writing:

$$
\left(\frac{1}{\left|Q_{\beta}^{k}\right|} \int_{Q_{\beta}^{k}}|f|^{p}\right)^{1 / p} \leq c_{n, p}\left(\frac{1}{\left|Q_{\beta}^{k}\right|} \int_{Q_{\beta}^{k}}\left(f_{\Omega_{n+2}, \Omega_{n+3}}^{\#}\right)^{p}\right)^{1 / p}
$$

(note that the local sharp function is $f_{\Omega_{n+2}, \Omega_{n+3}}^{\#}$ because we are using dyadic balls related to $\left.\Omega_{n+1}\right)$, that is

$$
\int_{Q_{\beta}^{k}}|f|^{p} \leq c_{n, p}^{p} \int_{Q_{\beta}^{k}}\left(f_{\Omega_{n+2}, \Omega_{n+3}}^{\#}\right)^{p} .
$$

Adding these inequalities for all the cubes $Q_{\beta}^{k}$ in $F_{\alpha}^{k}$ we get

$$
\begin{aligned}
\left(\int_{B\left(z_{\alpha}^{k}, c_{1} \delta^{k}\right)}|f|^{p}\right)^{1 / p} & \leq\left(\int_{F_{\alpha}^{k}}|f|^{p}\right)^{1 / p} \leq c_{n, p}\left(\int_{F_{\alpha}^{k}}\left(f_{\Omega_{n+2}, \Omega_{n+3}}^{\#}\right)^{p}\right)^{1 / p} \\
& \leq c_{n, p}\left(\int_{B\left(z_{\alpha}^{k}, c^{\prime} \delta^{k}\right)}\left(f_{\Omega_{n+2}, \Omega_{n+3}}^{\#}\right)^{p}\right)^{1 / p}
\end{aligned}
$$

which is our assertion, with $R=c_{1} \delta^{k}, \gamma=c^{\prime} / c_{1}$.

\section{Local $B M O$ and John-Nirenberg inequality}

We start defining the space of functions with locally bounded mean oscillation in a locally homogeneous space: if

Definition 4.1. Let $f \in L^{1}\left(\Omega_{n+1}\right)$. We say that $f$ belongs to $B M O\left(\Omega_{n}, \Omega_{n+1}\right)$

$$
[f]_{n} \equiv \sup _{x \in \Omega_{n}, r \leq 2 \epsilon_{n}} \frac{1}{\mu(B(x, r)} \int_{B(x, r)}\left|f(y)-f_{B(x, r)}\right| d \mu(y)<\infty .
$$

The main result in this section is the following.

Theorem 4.2. (Local John-Nirenberg inequality) There exist positive constants $b_{n}, R_{n}$ such that $\forall f \in B M O\left(\Omega_{n}, \Omega_{n+1}\right)$ and for any ball $B(a, R)$, with $a \in \Omega_{n}$ and $R \leq R_{n}$, the following inequality holds true

$$
\mu\left(\left\{x \in B(a, R):\left|f(x)-f_{B(a, R)}\right|>\lambda\right\}\right) \leq 2 e^{\frac{-b_{n} \lambda}{[f] n}} \mu(B(a, R)) \quad \forall \lambda>0 .
$$


Remark 4.3. As will appear from the proof, the constant $R_{n}$ is strictly smaller than the number $2 \varepsilon_{n}$ appearing in the definition of $B M O\left(\Omega_{n}, \Omega_{n+1}\right)$. Explicitly, we will see that

$$
R_{n}=\frac{2 \varepsilon_{n}}{B_{n+1}\left(\frac{9}{2} B_{n+1}^{2}+3 B_{n+1}+1\right)} .
$$

Proof. We can assume $[f]_{n}=1$, since (4.1) does not change dividing both $f$ and $\lambda$ for a constant. Let

$$
K_{n}=2 B_{n+1}+3 B_{n+1}^{2}
$$

be the constant appearing in Vitali covering Lemma 2.2,

$$
\alpha_{n}=B_{n+1}\left(\frac{3}{2} K_{n}+1\right), \quad R_{n}=\frac{2 \varepsilon_{n}}{\alpha_{n}} .
$$

Let $a \in \Omega_{n}, R \leq R_{n}$ and let $S=B(a, R)$ (since $R<\varepsilon_{n}, S \subseteq \Omega_{n+1}$ ). The proof consists in an iterative construction.

Step 1. We will prove that there exists a family of balls $\left\{S_{j}\right\}_{j=1}^{\infty} \subset S$ and constants $c, \lambda_{0} \geq 1$ depending on $n$ such that:

i) $\left\{x \in S:\left|f(x)-f_{S}\right|>\lambda_{0}\right\} \subset \bigcup_{j=1}^{\infty} S_{j} \subset S$;

ii) $\sum_{j=1}^{\infty} \mu\left(S_{j}\right) \leq \frac{1}{2} \mu(S)$;

iii) $\left|f_{S}-f_{S_{j}}\right| \leq c \lambda_{0}$.

To prove this we start defining the maximal operator associated to $S$ letting, for any $x \in S$,

$$
M_{S} f(x)=\sup \left\{\frac{1}{\mu(B)} \int_{B}\left|f(y)-f_{S}\right| d \mu(y): B \text { ball, } x \in B, B \subseteq \alpha_{n} S\right\}
$$

where $\alpha_{n} S=B\left(a, \alpha_{n} R\right) \subseteq \Omega_{n+1}$ since $\alpha_{n} R \leq 2 \varepsilon_{n}$.

We claim that there exists $A=A(n)>0$ such that for all $t>0$

$$
\mu\left(\left\{x \in S: M_{S} f(x)>t\right\}\right) \leq \frac{A}{t} \mu(S) .
$$

To show this, let $t>0$ and let

$$
U_{t}=\left\{x \in S: M_{S} f(x)>t\right\} .
$$

For every $x \in U_{t}$ there exists a ball $B_{x}$ such that $x \in B_{x} \subseteq \alpha_{n} S$ and

$$
\mu\left(B_{x}\right)<\frac{1}{t} \int_{B_{x}}\left|f(y)-f_{S}\right| d \mu .
$$

Now, by Vitali Lemma 2.7 there exists a countable subcollection of disjoint balls $\left\{B\left(x_{i}, r_{i}\right)\right\}$ such that

$$
U_{t} \subseteq \bigcup_{i=1}^{\infty} B\left(x_{i}, K_{n} r_{i}\right)
$$

Then, since by definition of $S$ and $M_{S} f, \cup_{i=1}^{\infty} B\left(x_{i}, r_{i}\right) \subseteq \alpha_{n} S \subset \Omega_{n+1}$, for some constant $A=A(n)$ which can vary from line to line we have

$$
\mu\left(U_{t}\right) \leq \mu\left(\bigcup_{i=1}^{\infty} B\left(x_{i}, K_{n} r_{i}\right)\right) \leq \sum_{i=1}^{\infty} \mu\left(B\left(x_{i}, K_{n} r_{i}\right)\right) \leq A \sum_{i=1}^{\infty} \mu\left(B\left(x_{i}, r_{i}\right)\right)
$$




$$
\begin{aligned}
& \leq \sum_{i=1}^{\infty} \frac{A}{t} \int_{B\left(x_{i}, r_{i}\right)}\left|f-f_{S}\right| d \mu=\frac{A}{t} \int_{\cup_{i=1}^{\infty} B\left(x_{i}, r_{i}\right)}\left|f-f_{S}\right| d \mu \\
& \leq \frac{A}{t} \int_{\alpha_{n} S}\left|f-f_{S}\right| d \mu \leq \frac{A}{t}\left\{\int_{\alpha_{n} S}\left|f-f_{\alpha_{n} S}\right| d \mu+\int_{\alpha_{n} S}\left|f_{\alpha_{n} S}-f_{S}\right| d \mu\right\} \\
& \leq \frac{A}{t}\left\{\mu\left(\alpha_{n} S\right)[f]_{n}+\mu\left(\alpha_{n} S\right)\left|f_{S}-f_{\alpha_{n} S}\right|\right\} \leq \frac{A}{t} \mu\left(\alpha_{n} S\right)[f]_{n} \leq \frac{A}{t} \mu(S)
\end{aligned}
$$

where we exploited the assumption $[f]_{n}=1$. Hence (4.3) is proved.

Let now $\lambda_{0}>A$, we consider the following open set

$$
U=\left\{x \in S: M_{S} f(x)>\lambda_{0}\right\} .
$$

We have, by (4.3),

$$
\mu(U \cap S)=\mu(U) \leq \frac{A}{\lambda_{0}} \mu(S)<\mu(S)
$$

from which

$$
S \cap U^{c} \neq \emptyset
$$

Then for any $x \in S$ we set

$$
r(x)=\frac{1}{2 K_{n}} \rho\left(x, U^{c}\right) \quad \forall x \in U .
$$

If $x, y \in S$ we have $\rho(x, y) \leq 2 B_{n+1} R$. Then $\forall x \in U$ (taking a point $y \in U^{c} \cap S$ in the following inequality)

$$
r(x) \leq \frac{1}{2 K_{n}} \rho(x, y) \leq \frac{1}{2 B_{n+1}\left(2+3 B_{n+1}\right)} 2 B_{n+1} R \leq \frac{R}{5} .
$$

If $y \in B\left(x, K_{n} r(x)\right)$ for some $x \in U$, we have

$$
\rho(y, x)<K_{n} r(x)=\frac{K_{n}}{2 K_{n}} \rho\left(x, U^{c}\right)<\rho\left(x, U^{c}\right)
$$

then $y \in U$, from which

$$
B\left(x, K_{n} r(x)\right) \subseteq U
$$

On the other hand

$$
U \subset \bigcup_{x \in U} B(x, r(x))
$$

and from the Vitali Lemma there exists a countable sequence of disjoint balls $\left\{B\left(x_{j}, r_{j}\right)\right\}$ $\left(r_{j}=r\left(x_{j}\right)\right)$ such that

$$
U \subset \bigcup_{j=1}^{\infty} B\left(x_{j}, K_{n} r_{j}\right)
$$

which by the inclusion (4.5) means that

$$
U=\bigcup_{j=1}^{\infty} B\left(x_{j}, K_{n} r_{j}\right) .
$$

Moreover, $B\left(x_{j}, 3 K_{n} r_{j}\right) \cap U^{c} \neq \emptyset \forall j \in \mathbf{N}$ and $B\left(x_{j}, 3 K_{n} r_{j}\right) \subseteq \alpha_{n} S$ since $\alpha_{n}=$ $B_{n+1}\left(\frac{3 K_{n}}{2}+1\right)$. 
If $y \in B\left(x_{j}, 3 K_{n} r_{j}\right) \cap U^{c}$, then $M_{s} f(y) \leq \lambda_{0}$ and

$$
\frac{1}{\mu\left(B\left(x_{j}, 3 K_{n} r_{j}\right)\right)} \int_{B\left(x_{j}, 3 K_{n} r_{j}\right)}\left|f-f_{S}\right| d \mu \leq \lambda_{0} .
$$

We now set

and we have, by (4.8)

$$
S_{j}=B\left(x_{j}, K_{n} r_{j}\right)
$$

$$
\begin{aligned}
\left|f_{S}-f_{S_{j}}\right| & =\left|\frac{1}{\mu\left(S_{j}\right)} \int_{S_{j}} f d \mu-f_{S}\right| \leq \frac{1}{\mu\left(S_{j}\right)} \int_{S_{j}}\left|f-f_{S}\right| d \mu \\
& \leq \frac{c}{\mu\left(B\left(x_{j}, 3 K_{n} r_{j}\right)\right)} \int_{B\left(x_{j}, 3 K_{n} r_{j}\right)}\left|f-f_{S}\right| d \mu \leq c \lambda_{0}
\end{aligned}
$$

which is point iii).

By the differentiation theorem we have that for a.e. $x \in S \backslash \cup_{j} S_{j}$ (that by (4.7) implies that $x \in U^{c}$ so that $\left.M_{S} f(x) \leq \lambda_{0}\right)$

$$
\left|f(x)-f_{S}\right| \leq \lambda_{0}
$$

This means that

$$
\left\{x \in S:\left|f(x)-f_{S}\right|>\lambda_{0}\right\} \subset \bigcup_{j=1}^{\infty} S_{j} \subset S
$$

which is point i). Moreover, by the doubling property (H7) and (4.4),

$$
\sum_{j=1}^{\infty} \mu\left(S_{j}\right) \leq c \sum_{j=1}^{\infty} \mu\left(B\left(x_{j}, r_{j}\right)\right)=c \mu\left(\bigcup_{j=1}^{\infty} B\left(x_{j}, r_{j}\right)\right) \leq c \mu(U) \leq c \frac{A}{\lambda_{0}} \mu(S)=\frac{1}{2} \mu(S),
$$

having finally chosen $\lambda_{0}=2 c A$, so that also point ii) is proved and step 1 is completed.

Step 2. We now do the same construction on each ball $S_{j}$ constructed in Step 1, which allows to conclude that, for every $j_{1}=1,2, \ldots$, there exists a sequence of balls $\left\{S_{j_{1} j_{2}}\right\}_{j_{2}=1}^{\infty} \subset S_{j_{1}}$ such that (for the same constants $c, \lambda_{0}$ of Step 1)

i) $\left\{x \in S_{j_{1}}:\left|f(x)-f_{S_{j_{1}}}\right|>\lambda_{0}\right\} \subset \bigcup_{j_{2}=1}^{\infty} S_{j_{1} j_{2}} \subset S_{j_{1}}$;

ii) $\sum_{j_{2}=1}^{\infty} \mu\left(S_{j_{1} j_{2}}\right) \leq \frac{1}{2} \mu\left(S_{j_{1}}\right)$;

iii) $\left|f_{S_{j_{1}}}-f_{S_{j_{1} j_{2}}}\right| \leq c \lambda_{0}$.

Point ii) of Step 2 and Step 1 imply

$$
\sum_{j_{1}, j_{2}=1}^{\infty} \mu\left(S_{j_{1} j_{2}}\right) \leq \frac{1}{2} \sum_{j_{1}=1}^{\infty} \mu\left(S_{j_{1}}\right) \leq \frac{1}{4} \mu(S)
$$

Also, point i) of Step 2 and point iii) of Step 1, imply that for a.e. $x \in S_{j_{1}} \backslash \bigcup_{j_{2}=1}^{\infty} S_{j_{1} j_{2}}$,

$$
\left|f(x)-f_{S}\right| \leq\left|f(x)-f_{S_{j_{1}}}\right|+\left|f_{S_{j_{1}}}-f_{S}\right| \leq \lambda_{0}+c \lambda_{0}<2 c \lambda_{0}
$$

which means that

$$
\left\{x \in S_{j_{1}}:\left|f(x)-f_{S}\right|>2 c \lambda_{0}\right\} \subset \bigcup_{j_{2}=1}^{\infty} S_{j_{1} j_{2}} \subset S_{j_{1}} .
$$


However, point i) of Step 1 implies

$$
\left\{x \in S:\left|f(x)-f_{S}\right|>2 c \lambda_{0}\right\} \subset\left\{x \in S:\left|f(x)-f_{S}\right|>\lambda_{0}\right\} \subset \bigcup_{j_{1}=1}^{\infty} S_{j_{1}}
$$

hence (4.10) rewrites as

$$
\left\{x \in S:\left|f(x)-f_{S}\right|>2 c \lambda_{0}\right\} \subset \bigcup_{j_{1}, j_{2}=1}^{\infty} S_{j_{1} j_{2}}
$$

and, letting $\lambda_{1}=c \lambda_{0}$,

$$
\mu\left(\left\{x \in S:\left|f(x)-f_{S}\right|>2 \lambda_{1}\right\}\right) \leq \frac{1}{4} \mu(S) .
$$

Relation (4.11) summarizes the joint consequences of Steps 1 and 2.

Proceeding this way the iterative construction, at Step $N$ we will have that

$$
\mu\left(\left\{x \in S:\left|f(x)-f_{S}\right|>N \lambda_{1}\right\}\right) \leq \frac{1}{2^{N}} \mu(S) .
$$

Now, let $\lambda>0$. If $\lambda \geq \lambda_{1}$, let $N$ be the positive integer such that

$$
N \lambda_{1}<\lambda \leq(N+1) \lambda_{1},
$$

then

$$
\begin{aligned}
\mu\left(\left\{x \in S:\left|f(x)-f_{S}\right|>\lambda\right\}\right) & \leq \mu\left(\left\{x \in S:\left|f(x)-f_{S}\right|>N \lambda_{1}\right\}\right) \\
& \leq \frac{1}{2^{N}} \mu(S)=e^{-N \log 2} \mu(S) \leq 2 e^{-\left(\frac{\log 2}{\lambda_{1}}\right) \lambda} \mu(S) .
\end{aligned}
$$

Finally, if $0<\lambda \leq \lambda_{1}$,

$$
\mu\left(\left\{x \in S:\left|f(x)-f_{S}\right|>\lambda\right\}\right) \leq \mu(S) \leq 2 e^{-\left(\frac{\log 2}{\lambda_{1}}\right) \lambda} \mu(S)
$$

and the assertion follows (recall we are assuming $[f]_{n}=1$ ), with $b_{n}=\frac{\log 2}{\lambda_{1}}$.

Definition 4.4. Let $p \in(1,+\infty)$. We say that $f$ belongs to $B M O^{p}\left(\Omega_{n}, \Omega_{n+1}\right)$ if $f \in L^{p}\left(\Omega_{n+1}\right)$ and

$$
[f]_{p, n} \equiv \sup _{x \in \Omega_{n}, r \leq R_{n}}\left(\frac{1}{\mu(B(x, r))} \int_{B(x, r)}\left|f(y)-f_{B(x, r)}\right|^{p} d \mu(y)\right)^{1 / p}<\infty
$$

where $R_{n}$ is the constant appearing in (4.2), strictly smaller than $2 \varepsilon_{n}$.

Now we compare the spaces $B M O\left(\Omega_{n}, \Omega_{n+1}\right)$ and $B M O^{p}\left(\Omega_{n}, \Omega_{n+1}\right)$.

Theorem 4.5. For any $p \in(1, \infty)$ and $n$ we have

$$
B M O^{p}\left(\Omega_{n}, \Omega_{n+1}\right)=B M O\left(\Omega_{n}, \Omega_{n+1}\right) .
$$

Moreover, there exists a positive constant $c_{n, p}$ such that for any $f \in B M O\left(\Omega_{n}, \Omega_{n+1}\right)$,

$$
[f]_{p, n} \leq c_{n, p}[f]_{n} .
$$

In particular, $B M O\left(\Omega_{n}, \Omega_{n+1}\right) \subseteq L^{p}\left(\Omega_{n}\right)$ for every $p \in(1, \infty)$.

Remark 4.6. Comparing this result with those about the local Fefferman-Stein function proved in the previous section (for instance, Corollary 3.9), we see that the present theorem is a "local" result in a different sense. Here, in the upper bound (4.13), there is not an enlargement of the domain, passing from the left to the right hand side; instead, the local seminorms $[f]_{p, n}$ are computed taking the supremum 
over balls of radii $r \leq R_{n}$, which is a stricter condition than the bound $r \leq 2 \varepsilon_{n}$ defining the seminorm $[f]_{n}$.

Proof. Let $f \in B M O^{p}\left(\Omega_{n}, \Omega_{n+1}\right)$. By Hölder's inequality we can write, for every $x \in \Omega_{n}, r \leq R_{n}$,

$$
\begin{aligned}
& \frac{1}{\mu(B(x, r))} \int_{B(x, r)}\left|f(y)-f_{B(x, r)}\right| d \mu(y) \\
& \leq\left(\frac{1}{\mu(B(x, r))} \int_{B(x, r)}\left|f(y)-f_{B(x, r)}\right|^{p} d \mu(y)\right)^{1 / p} \leq[f]_{p, n}<\infty .
\end{aligned}
$$

On the other hand, if $R_{n}<r<2 \varepsilon_{n}$ we have

$$
\begin{aligned}
& \frac{1}{\mu(B(x, r))} \int_{B(x, r)}\left|f(y)-f_{B(x, r)}\right| d \mu(y) \leq 2 \frac{1}{\mu(B(x, r))} \int_{B(x, r)}|f(y)| d \mu(y) \\
& \leq 2 \frac{1}{\mu\left(B\left(x, R_{n}\right)\right)} \int_{\Omega_{n+1}}|f(y)| d \mu(y) \leq c_{n}\|f\|_{L^{p}\left(\Omega_{n+1}\right)}
\end{aligned}
$$

because

$$
\inf _{x \in \Omega_{n}} \mu\left(B\left(x, R_{n}\right)\right) \geq c_{n}>0
$$

as can be easily proved as a consequence of the local doubling condition. Therefore $[f]_{n}<\infty$ and $f \in B M O\left(\Omega_{n}, \Omega_{n+1}\right)$.

Conversely, to prove (4.13), let $B$ be a ball centered in $x \in \Omega_{n}$ with radius $r \leq R_{n}$. Then by Theorem 4.2 we have

$$
\begin{aligned}
\int_{B}\left|f(y)-f_{B}\right|^{p} d \mu(y) & =\int_{0}^{+\infty} p \lambda^{p-1} \mu\left(\left\{z \in B:\left|f(z)-f_{B}\right|>\lambda\right\}\right) d \lambda \\
& \leq 2 \int_{0}^{+\infty} p \lambda^{p-1} e^{-b_{n} \lambda /[f]_{n}} \mu(B) d \lambda \\
& =\mu(B)[f]_{n}^{p} 2 p \int_{0}^{+\infty} t^{p-1} e^{-b_{n} t} d t
\end{aligned}
$$

from which

$$
\left(\frac{1}{\mu(B)} \int_{B}\left|f(y)-f_{B}\right|^{p} d \mu(y)\right)^{1 / p} \leq\left(2 p \int_{0}^{+\infty} t^{p-1} e^{-b_{n} t} d t\right)^{1 / p}[f]_{n}=c_{n, p}[f]_{n}
$$

which gives (4.13) and the inclusion $B M O\left(\Omega_{n}, \Omega_{n+1}\right) \subseteq B M O^{p}\left(\Omega_{n}, \Omega_{n+1}\right)$.

Finally, to show that $f \in L^{p}\left(\Omega_{n}\right)$ we can cover $\Omega_{n}$ with a finite collection of balls $B\left(x, R_{n}\right)$ with $x \in \Omega_{n}$, writing

$$
\begin{aligned}
\left(\frac{1}{\mu(B)} \int_{B}|f(y)|^{p} d \mu(y)\right)^{1 / p} & \leq\left(\frac{1}{\mu(B)} \int_{B}\left|f(y)-f_{B}\right|^{p} d \mu(y)\right)^{1 / p}+\left|f_{B}\right| \\
& \leq[f]_{p, n}+\frac{1}{\mu(B)}\|f\|_{L^{1}\left(\Omega_{n+1}\right)}<\infty
\end{aligned}
$$

which implies the finiteness of $\|f\|_{L^{p}\left(\Omega_{n}\right)}$.

\section{References}

[1] Bramanti, M.: Singular integrals in nonhomogeneous spaces: $L^{2}$ and $L^{p}$ continuity from Hölder estimates. - Rev. Mat. Iberoam. 26:1, 2010, 347-366.

[2] Bramanti, M., G. Cupini, E. Lanconelli, and E. Priola: Global $L^{p}$ estimates for degenerate Ornstein-Uhlenbeck operators. - Math. Z. 266:4, 2010, 789-816. 
[3] Bramanti, M., and M. Zhu: Local real analysis in locally homogeneous spaces. - Manuscripta Math. 138:3-4, 2012, 477-528.

[4] Bramanti, M., and M. Toschi: The sharp maximal function approach to $L^{p}$ estimates for operators structured on Hörmander's vector fields. - Rev. Mat. Complut. 29, 2016, 531-557.

[5] Bramanti, M., and M. Zhu: $L^{p}$ and Schauder estimates for nonvariational operators structured on Hörmander vector fields with drift. - Anal. PDE 6:8, 2013, 1793-1855.

[6] BuCkLey, S. M.: Inequalities of John-Nirenberg type in doubling spaces. - J. Anal. Math. 79, $1999,215-240$.

[7] Carbery, A., J. VAnce, S. Wainger, and J. Wright: A variant of the notion of a space of homogeneous type. - J. Funct. Anal. 132:1, 1995, 119-140.

[8] Caruso, A. O., and M. S. FAnciullo: $B M O$ on spaces of homogeneous type: a density result on C-C spaces. - Ann. Acad. Sci. Fenn. Math. 32, 2007, 13-26.

[9] Castillo, R. E., J. C. Ramos Fernández, and E. Trousselot: Functions of bounded $(\varphi, p)$ mean oscillation. - Proyecciones 27:2, 2008, 163-177.

[10] Christ, M.: A $T(b)$ theorem with remarks on analytic capacity and the Cauchy integral. Colloq. Math. 60/61:2, 1990, 601-628.

[11] Colfman, R. R., and G. Weiss: Analyse harmonique non-commutative sur certains espaces homogènes. - Lecture Notes in Math. 242, Springer-Verlag, Berlin-New York, 1971.

[12] DAfni, G., and H. YuE: Some characterizations of local bmo and $h^{1}$ on metric measure spaces. - Anal. Math. Phys. 2:3, 2012, 285-318.

[13] Diening, L., M. RŮŽIČKa, and K. Schumacher: A decomposition technique for John domains. - Ann. Acad. Sci. Fenn. Math. 35:1, 2010, 87-114.

[14] Fefferman, C., and E. M. Stein: $H^{p}$ spaces of several variables. - Acta Math. 129:3-4, 1972, $137-193$.

[15] Grafakos, L., L. LiU, and D. YAnG: Vector-valued singular integrals and maximal functions on spaces of homogeneous type. - Math. Scand. 104:2, 2009, 296-310.

[16] IwAniec, T.: On $L^{p}$-integrability in PDEs and quasiregular mappings for large exponents. Ann. Acad. Sci. Fenn. Ser. A I Math. 7:2, 1982, 301-322.

[17] JaWerth, B., and A. Torchinsky: Local sharp maximal functions. - J. Approx. Theory 43:3, $1985,231-270$.

[18] John, F., and L. Nirenberg: On functions of bounded mean oscillation. - Comm. Pure Appl. Math. 14, 1961, 415-426.

[19] Kronz, M.: Some function spaces on spaces of homogeneous type. - Manuscripta Math. 106:2, 2001, 219-248.

[20] Krylov, N. V.: Parabolic and elliptic equations with VMO coefficients. - Comm. Partial Differential Equations 32:1-3, 2007, 453-475.

[21] LAI, Q.: The sharp maximal function on spaces of generalized homogeneous type. - J. Funct. Anal. 150:1, 1997, 75-100.

[22] Mateu, J., P. Mattila, A. Nicolau, and J. Orobitg: BMO for nondoubling measures. Duke Math. J. 102:3, 2000, 533-565.

[23] Pradolini, G., and O. Salinas: Commutators of singular integrals on spaces of homogeneous type. - Czechoslovak Math. J. 57(132):1, 2007, 75-93.

[24] Shi, X. L., and A. Torchinsky: Local sharp maximal functions in spaces of homogeneous type. - Sci. Sinica Ser. A 30:5, 1987, 473-480. 